\title{
Erratum to: Fluorescence Quenching to Study Protein-ligand Binding: Common Errors
}

\author{
Marco van de Weert
}

Received: 9 August 2011 / Accepted: 10 August 2011 /Published online: 27 August 2011

(C) Springer Science+Business Media, LLC 2011

Erratum to: J Fluoresc (2010) 20:625-629

$$
\text { DOI 10.1007/s10895-009-0572-x }
$$

The author regrets that there were some factual errors in the paper, corrected below. These errors do not minimise the criticism contained in the paper, but rather raise further issues with a significant number of papers using fluorescence quenching to characterise ligand binding.

1. On page 628, in the section on Dynamic Quenching, it is stated that rejection of collisional quenching means the quenching is static. Later, pitfalls 5 and 7 discuss static quenching due to structural changes and FRET, respectively. This is unfortunately incorrect, as FRET is by definition a dynamic quenching mechanism [1], while (de)quenching due to structural changes are also generally dynamic in nature. These sections can be corrected by exchanging "dynamic quenching" with "collisional quenching" and "static quenching" with "binding-related quenching".

2. In the derivation of equation 6 , the implicit and unmentioned assumption is made that the binding is infinitely cooperative. Such strong cooperativity is very uncommon in nature and thus generally an invalid assumption. Thus, even when correcting for the pitfalls discussed in the original paper, this equation is unlikely to yield the true number of binding sites, and returns a value (the Hill coefficient) that only indicates the presence or absence of cooperativity between binding sites (see also [2, 3]).

The two issues above have been correctly described in a recent and more extensive criticism by the author [3]. Once again the author apologises for any confusion that may have been caused.

\section{References}

1. Braslavsky SE (2007) Glossary of terms used in photochemistry, 3rd edition (IUPAC Recommendations 2006). Pure Appl Chem 79:293-465

2. Lissi E, Abuin E (2011) On the evaluation of the number of binding sites in proteins from steady state fluorescence measurements. J Fluoresc. doi:10.1007/s10895-011-0887-2-

3. van de Weert M, Stella L (2011) Fluorescence quenching and ligand binding: a critical discussion of a popular methodology. J Mol Struct 998:144-150

The online version of the original article can be found at http://dx.doi. org/10.1007/s10895-009-0572-x.

\footnotetext{
M. van de Weert $(\bowtie)$

Department of Pharmaceutics and Analytical Chemistry, Faculty

of Pharmaceutical Sciences, University of Copenhagen,

Universitetsparken 2,

DK-2100, Copenhagen, Denmark

e-mail: mvdw@farma.ku.dk
} 